\title{
Racial and Ethnic Differences in Left Atrial Appendage Occlusion Wait Time, Complications, and Periprocedural Management
}

\author{
Abdullah Haddad ${ }^{1}$, Matthew Bocchese ${ }^{1}$, Rebecca Garber ${ }^{1}$, Brian O’Neill², George \\ Yesenosky $^{1}$, Pravin Patil ${ }^{1}$, Martin Keane ${ }^{1}$, Sabrina Islam ${ }^{1}$, Jacqueline Sherrer ${ }^{1}$, Anuj \\ Basil $^{1}$, Chethan Gangireddy ${ }^{1}$, Joshua Cooper ${ }^{1}$, Edmond Cronin ${ }^{1}$, and Isaac Whitman ${ }^{1}$ \\ ${ }^{1}$ Temple University Hospital \\ ${ }^{2}$ Henry Ford Health System
}

August 24, 2020

\begin{abstract}
Introduction: Non-white patients are underrepresented in left atrial appendage occlusion (LAAO) trials, and racial disparities in LAAO periprocedural management are unknown. Methods: We assessed sociodemographics and comorbidities of consecutive patients at our institution undergoing LAAO between 2015 - 2020, then in adjusted analyses, compared procedural wait time, procedural complications, and post-procedure oral anticoagulation (OAC) use in whites versus non-whites. Results: Among 109 patients undergoing LAAO ( $45 \%$ white), whites were less likely to have heart failure than non-whites $(18 \%$ vs. $48 \%, \mathrm{p}=0.001)$, prior stroke ( $14 \%$ vs. $43 \%, \mathrm{p}=0.001)$, or end stage renal disease ( 0 vs. $20 \%, \mathrm{p}<0.001)$. Whites had lower CHA2DS2VASc scores, on average ( 4.0 vs. $4.8, \mathrm{p}=0.006)$. There was no difference in median time from index event or initial outpatient cardiology encounter to LAAO procedure (whites 10.5 vs. non-whites 13.7 months, $\mathrm{p}=0.9 ; 1.9$ vs 1.8 months, $\mathrm{p}=0.6$, respectively), and there was no difference in procedural complications (whites $4 \%$ vs. non-whites $5 \%, \mathrm{p}=0.33$ ). After adjusting for CHA2DS2VASc score, OAC use at discharge tended to be higher in whites (OR 2.4, 95\% CI [0.9-6.0], $\mathrm{p}=0.07$ ). When restricting the analysis to those with prior gastrointestinal (GI) bleed, adjusting for CHA2DS2VASc score and GI bleed severity, whites had a nearly five-fold odds of being discharged on OAC (OR 4.6, 95\% CI [1-21.8], $\mathrm{p}=0.05$ ). Conclusion: Despite an increased prevalence of comorbidities amongst non-whites, wait time for LAAO and procedural complications were similar in whites versus non-whites. Among those with prior GI bleed, whites were nearly five-fold more likely to be discharged on OAC than non-whites.
\end{abstract}

\section{Hosted file}

Watchman Manuscript JCE.pdf available at https://authorea.com/users/353322/articles/ 477279-racial-and-ethnic-differences-in-left-atrial-appendage-occlusion-wait-timecomplications-and-periprocedural-management 